\title{
A WIDE-BAND ELECTROMAGNETIC IMPEDANCE PROFILING SYSTEM FOR NON-INVASIVE SUBSURFACE CHARACTERIZATION
}

\author{
Hung-Wen Tseng, Lawrence Berkeley National Laboratory, Berkeley, CA \\ Ki Ha Lee, Lawrence Berkeley National Laboratory, Berkeley, CA \\ Alex Becker, University of California at Berkeley, Berkeley, CA
}

\begin{abstract}
A non-invasive, wide-band electromagnetic (EM) impedance difference system for shallow subsurface electrical structure characterization in environmental and engineering problems has been developed at the Lawrence Berkeley National Laboratory (LBNL). Electrical parameters of interest are electrical conductivity and dielectric permittivity that are deduced from the impedance difference data. The prototype system includes a magnetic loop transmitter, which operates between $0.1 \mathrm{MHz}$ and 100 $\mathrm{MHz}$, an electrical dipole antenna for observing the electric field, and a loop antenna for measuring the magnetic field. All antennas are mounted on a cart made of non-metallic material for easy movement of the whole array for profiling. Surface EM impedance difference is obtained by taking the difference of the ratios of the electric fields to the magnetic fields at selected frequencies at two different levels. Numerical simulations verify this approach. A set of the impedance difference data acquired at the University of California's Richmond Field Station compares reasonably well with simulation results based on a model obtained with the resistivity method and in situ TDR (time domain reflectometry) measurements.
\end{abstract}

\section{Introduction}

Accurate information on the shallow $(<10 \mathrm{~m})$ subsurface distribution in electrical conductivity and dielectric permittivity, which are related to local geochemistry and geohydrology, is useful for delineation of buried waste, detection of unexploded ordinance, verification and monitoring of containment distributions, and other environmental applications. Electromagnetic (EM) measurements at frequencies up to $100 \mathrm{MHz}$ are critical for such applications because the ability to determine the dielectric permittivity in addition to electrical conductivity of the subsurface is possible. Stewart et al. (1994) reviewed the importance of utilizing EM techniques with frequencies higher than $300 \mathrm{kHz}$ for obtaining the two subsurface electrical properties. One of the conclusions was that frequencies beyond $50 \mathrm{MHz}$ were required for better subsurface characterizations.

A prototype non-invasive, wide-band EM impedance system for near surface electrical structure characterization has been developed at the Lawrence Berkeley National Laboratory first by Frangos (2000) and then upgraded by Tseng et al. (2003b). The system can be operated at any selected frequency between 0.1 and $100 \mathrm{MHz}$. Since the full waveform of the EM fields is measured, antenna calibration plays a critical role for successful data acquisition. A related algorithm for 1-D inversion of the impedance data has also been explored to obtain the ground electrical conductivity and permittivity distributions (Song et al., 2002). In that study, however, plane wave incidence is assumed by placing the EM source far away from the receiving sensors.

Since the completion of the upgraded system, it has been found that the difference in the impedance data at two heights can also be used to infer the two electrical parameters. More importantly, using differenced data greatly decreases the difficulty in obtaining accurate antenna factors. Our intention is therefore to explore the feasibility of using this new approach for near-surface surveys. In 
this paper, the system described briefly and simulation data are presented to demonstrate responses of the system to various models. A newly developed algorithm for inverting the impedance difference data for both subsurface 1-D conductivity and permittivity is presented. Then field data is compared with simulation data based on a model derived from a resistivity sounding and an in situ TDR (time domain reflectometry) measurement.

\section{High frequency impedance difference and numerical simulations}

The impedance system, which is detailed by Tseng et al. (2003b) and depicted in Figure 1, consists of four major components: a controlling/recording unit, a loop magnetic dipole used as an EM field source, an electrical dipole and a loop magnetic antenna used to measure the electric and the magnetic fields, respectively, due to the transmitter. Based on previous study (Frangos, 2000) on the sensitivity of surface impedance to underground electrical structures, the magnetic dipole source is oriented horizontally in the preset y-direction while a radial horizontal electrical field (Ex) and a horizontal magnetic field $\left(\mathrm{H}_{\mathrm{y}}\right)$ are measured. Currently, all antennas are placed at a height of $1 \mathrm{~m}$ and are mounted on a manually towable cart for easy mobilization of the whole array. The transmitterreceiver separation can vary from $5.6 \mathrm{~m}$ to $10 \mathrm{~m}$. In cases in which larger separations are required, the antennas are mounted on three tripods. The control unit, which is composed of a laptop computer and a network analyzer, sends a continuous sinusoidal waveform at a preset frequency to the transmitter antenna via an optical link to be amplified into current flowing in the loop. The EM fields received by the two receiving antennas are transmitted as modulated optical signals to the network analyzer for recording and stacking. The ratio between the measured electric and the magnetic fields $\left(\mathrm{E}_{\mathrm{x}} / \mathrm{H}_{\mathrm{y}}\right)$ is calculated to obtain the impedance, which is used to invert for ground electrical conductivity and permittivity. Such survey technique is, in some sense, similar to a magneto-telluric (MT) sounding except that the source is operated at much higher frequencies and is much closer to the receiving antennas so that the plane wave field assumption for the MT survey is not valid here. To obtain the difference in impedance data, another reading is taken at a different level (about $0.3 \mathrm{~m}$ lower than the original one).

To demonstrate the response of the impedance difference system to subsurface electrical properties, several models are postulated and calculated with a 1-D algorithm developed at LBNL (Seol et al., 2002). The first model, depicted in Figure 2(a) and simulating a moderately dry soil layer, is a homogeneous half space with a conductivity of $0.01 \mathrm{~S} / \mathrm{m}$ and a relative dielectric constant of 6 . The transmitter-receiver separation is $5.6 \mathrm{~m}$ and antennas are at a height of $1 \mathrm{~m}$. The blue lines in Figure 2(b) represent the real and the imaginary parts of the calculated impedance differences for the half space from 0.1 to $100 \mathrm{MHz}$. For this half space model, GPR is of little use since there is no dielectric contrast in the ground to produce radar reflection signals.

A more conductive $0.1 \mathrm{~S} / \mathrm{m}$ layer is added to the half space between 2 and $3 \mathrm{~m}$ in depth to form a three-layered model labeled as MODEL 1 in Figure 2(a). Relative dielectric constants are set to 6 for all layers. The corresponding impedance difference is presented as red dots in Figure 2(b). In comparison, the real part of the impedance difference for MODEL 1 deviates considerably from that of the half space for frequencies below $4 \mathrm{MHz}$. Another layer, with relative dielectric constant of 25, is added to MODEL 1 between 1 and $2 \mathrm{~m}$ in depth and is labeled as MODEL 2 in Figure 2(a). Its impedance difference, shown in green lines in Figure 2(b), differs from that of MODEL 1 in the imaginary part for frequencies higher than $15 \mathrm{MHz}$. A two-layered model, MODEL 3 in Figure 2(a), has a $0.2 \mathrm{~m}$ thin layer with a relative dielectric constant of 25 on top of a half space with a relative permittivity of 6 . Conductivities of both layers are set to $0.01 \mathrm{~S} / \mathrm{m}$. The pattern of the impedance difference, shown as black dots in Figure 2(b), for this model is quite distinct from all others in both real and imaginary components above $15 \mathrm{MHz}$. 


\section{1-D inversion using the impedance difference data}

Several computer algorithms for interpreting surface-based high frequency EM data to obtain subsurface electrical conductivity and permittivity in a 1-D sense have been studied in the last decade. Stewart et al. (1994) used tilt angles and ellipticities of magnetic fields alone between $300 \mathrm{kHz}$ and 30 MHz. Kim et al. (1997) developed a code for inverting two-layered Cole-Cole models using magnetic fields for frequencies between 0.1 and $50 \mathrm{MHz}$. Based on the plane wave incidence approach, Song et al. (2002) used impedance data between 0.1 to $100 \mathrm{MHz}$ to invert for three-layered electrical properties. Due to the proximity of the receiving antennas to the EM source, the plane wave assumption does not apply to this study. Considering stability of the inversion processes to obtain reasonable subsurface structures, we have developed an algorithm for 1-D inversion of the impedance difference data based on a non-linear least squared approach similar to the Occam's inversion described by Constable et al. (1987).

The inversion starts with setting up an object functional, $\phi$, defined as

$$
\left.\phi=\| \underline{\underline{\mathbf{W}_{d}}} \underline{\underline{\mathbf{d}}}-\underline{\mathbf{d}}^{o b s}\right)\left\|^{2}+\lambda\right\| \underline{\underline{\mathbf{W}_{m}}} \underline{\underline{\mathbf{m}}} \|^{2}
$$

Here, $\underline{\mathbf{d}}$ and $\underline{\mathbf{d}}^{o b s}$ are the calculated impedance difference and observed data, respectively, $\mathbf{W}_{d}$ is a data weighting matrix usually its diagonals are the inverse of the noise level of corresponding data, $\underline{\mathbf{m}}$ represents the 1-D conductivity $(\underline{\sigma})$ and dielectric permittivity $(\underline{\varepsilon})$ of each layer, and ${\underline{\underline{\mathbf{W}_{m}}}}_{\text {is a model }}$ weighting matrix which determines how the model is smoothed. In this study, the difference of the model parameters, i.e., its first derivative, is used for the smoothness criteria. The Lagrange multiplier, $\lambda$, is used to control the degree of smoothness of the model. Minimizing the object functional with respect to $\underline{m}$, a system matrix can be derived and used to obtain an updated model for next iteration:

$$
\left.\left(\underline{\underline{\mathbf{J}}}^{T} \underline{\underline{\mathbf{W}_{d}}} \underline{\underline{\underline{\mathbf{W}_{d}}}} \underline{\underline{\mathbf{J}}}+\lambda \underline{\underline{\mathbf{W}_{m}}} \underline{\underline{\mathbf{W}_{m}}}\right) \underline{\mathbf{m}}^{k+1}=\underline{\underline{\mathbf{J}}}^{T} \underline{\underline{\mathbf{W}_{d}}} \underline{\underline{\mathbf{W}_{d}}} \underline{\underline{\mathbf{J}}} \underline{\underline{\mathbf{m}}}^{k}-\underline{\mathbf{d}}\left(\underline{\mathbf{m}}^{k}\right)+\underline{\mathbf{d}}^{o b s}\right) \text {. }
$$

Here, $\underline{\underline{\mathbf{J}}}$ is the Jacobian matrix, which is the change in impedance difference with respect to model parameter perturbation. The superscripts $T$ and $k$ represent the matrix transpose and the number of iteration, respectively. Starting from an initial model, usually a homogeneous half space, model parameters are updated for each iteration till the object functional reaches a preset value which typically is representative of noise level contained in the data. The Lagrange multiplier is assumed a large value at the beginning of the inversion and is reduced by half for the next successful iteration (Tseng et. al., 2003a).

To demonstrate the inversion algorithm, the simulated impedance difference data from MODEL2 (shown in Figure 2(b)) is inverted. Initially, both conductivity and dielectric constant are simultaneously inverted for but it was found that the inversion could not correctly recover the electrical properties of MODEL 2 as shown in Figure 2(a). This may be due to the impedance difference being much more sensitive to the conductivity than to the permittivity. To overcome this problem, the optimization process is completed in two steps: invert for conductivity only with low frequency data and then for permittivity with high frequency data. In this case, 12 frequencies from 0.1 to $2 \mathrm{MHz}$ were selected to recover the 1-D conductivity model, which is divided into 16 layers overlaying a homogeneous half space. The thickness of each layer is set to $0.5 \mathrm{~m}$ with an initial conductivity of $0.01 \mathrm{~S} / \mathrm{m}$ while dielectric constants of all layers were set to 6. This tactic is reasonable since the effect of the dielectric constant of subsurface material is negligible at the selected low-frequency range. Such an approach is also supported by the simulation data displayed in Figure 2 for this model. Presented in Figure 3(a) is 
the inverted 1-D conductivity distribution, which will be fixed afterwards when the permittivity distribution is inverted for. Due to the model smoothness constraint applied to the inversion, the conductivity section is smeared with respect to depth. However, the conductivity peaks at the same depth as the simulated MODEL 2, which is also plotted in red in Figure 3(a). For the second step, considering the rapid spatial change of the impedance difference with respect to the dielectric constant for frequencies higher than $10 \mathrm{MHz}$, the 1-D model is logarithmically divided into 21 layers, including the bottom half space. Twelve frequencies between 10 to $60 \mathrm{MHz}$ were chosen and the final result is shown in Figure 3(b). Though a minor spurious layer exists at about $0.3 \mathrm{~m}$ in depth, the target permittivity anomaly can be well identified between 1 and $2 \mathrm{~m}$.

\section{Field data example}

A set of impedance difference data has been acquired for demonstration at the University of California's Richmond Field Station, which is located in a geologically alluvial environment. The transmitter and receivers were separated by $6 \mathrm{~m}$. The amplitude and phase of the measured impedance difference are presented in Figure 4(a) and marked as blue asterisks. A resistivity sounding and a set of in situ TDR measurement were made to get the very near surface electrical properties, which are shown in Figure 4(b). Calculated impedance difference based on the section is calculated and is marked in red in Figure 4(a). While data below $300 \mathrm{kHz}$ are severely contaminated by noise, measured data matches model calculation reasonably well up to $40 \mathrm{MHz}$. The mismatch at frequencies above $40 \mathrm{MHz}$ may be due to noise and the interpreted resistivity model may not represent the real ground electrical structure. The inversion scheme described previously will be used to interpret the difference data.

\section{Summary}

High-frequency EM impedance difference is a new approach to characterize near-surface conductivity and permittivity. Here, feasibility of the method has been shown, for both the data acquisition system and the interpretation of acquired data. Comparison of field data with numerical simulations, which is based on a model derived from resistivity sounding and TDR measurements, also confirms that the prototype impedance system is working properly and is capable of delivering data as required. However, noise reduction and antenna calibration still remain critical for practical data acquisition and interpretation.

\section{References:}

Constable, S. C., Parker, R. L., and Constable, C. G., 1987, Occam's inversion: A practical algorithm for generating smooth models from electromagnetic sounding data: Geophysics, 52, 289-300.

Frangos, W., 2000, High Frequency Impedance Measurements for Non-invasive Permittivity Determination: PhD Thesis, University of California, Berkeley.

Kim, H. J., Song, Y., and Lee, K. H., 1997, High-frequency electromagnetic inversion for a depressive layered earth: J. Geomag. Geoelectr., 49, 1439-1450.

Seol, S. J., Suh, J. H., Song, Y., Kim, H. J., and Lee, K. H., 2002, The potential of fracture imaging using high-frequency, single-hole electromagnetic data: Geophysics, 67, 1087-1094.

Song, Y., Kim, H. J., and Lee, K.H., 2002, High-frequency EM impedance method for subsurface imaging: Geophysics, 67, 501-510. 
Stewart, D. C., Anderson, W. L., Grover, T. P., and Labson, V. F., 1994, Shallow subsurface mapping by electromagnetic sounding in the $300 \mathrm{kHz}$ to $30 \mathrm{MHz}$ range: Model studies and prototype system assessment: Geophysics, 59, 1201-1210.

Tseng, H.-W., Lee, K. H., and Becker, A., 2003a, 3D interpretation of electromagnetic data using a modified extended Born approximation: Geophysics, 68, 127-137.

Tseng, H.-W., Lee, K. H., and Becker, A., 2003b, A high frequency electromagnetic impedance imaging system: Proceedings, Symposium on the Application of Geophysics to Engineering and Environmental Problems, 534-540.

\section{Acknowledgements}

This work is supported by the Assistant Secretary for Environmental Restoration and Waste Management, Office of Technology Development of the U.S. Department of Energy under contract No. DE-AC03-76SF00098.

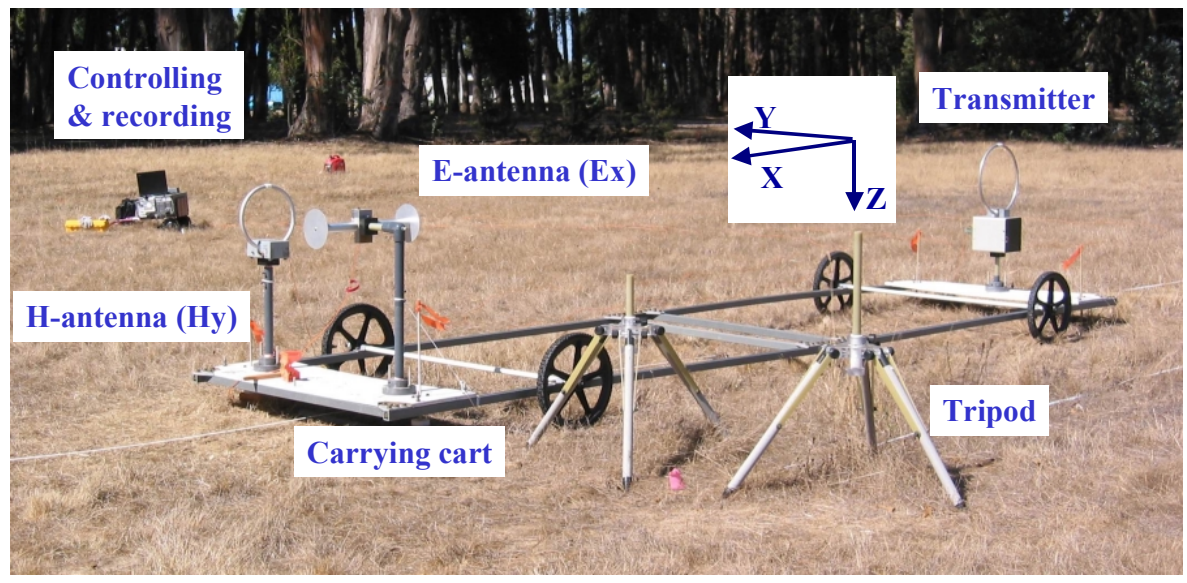

Fig. 1 Field setup of the high frequency impedance system. Transmitter-receiver separation is $5.6 \mathrm{~m}$ and all antennas are at $1 \mathrm{~m}$ height. 
(a)

$$
\begin{aligned}
& \text { Half-space }
\end{aligned}
$$

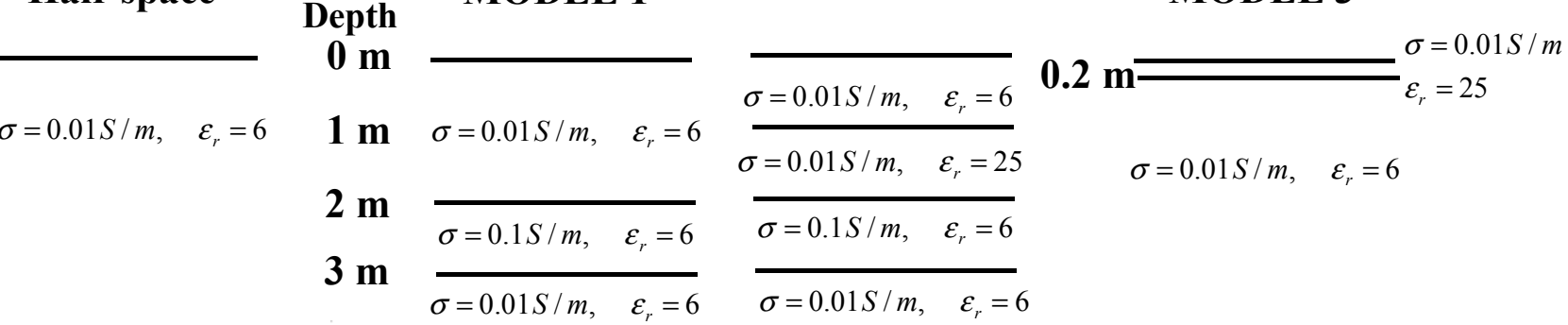

(b)
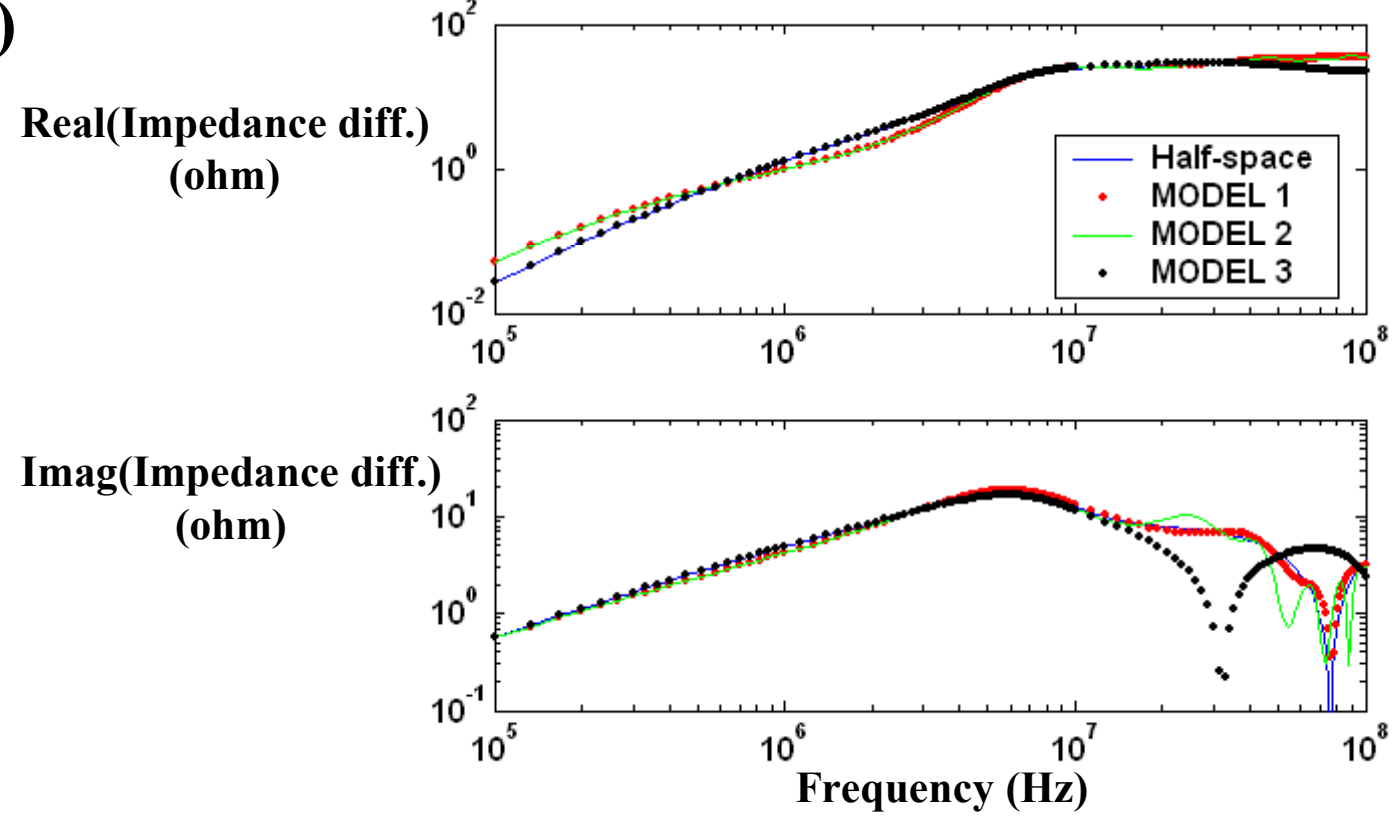

Fig. 2 (a) Simulated models to show impedance differences with respect to various 1-D electrical structures. (b) Calculated impedance differences vs. frequency for the four models described in (a). 

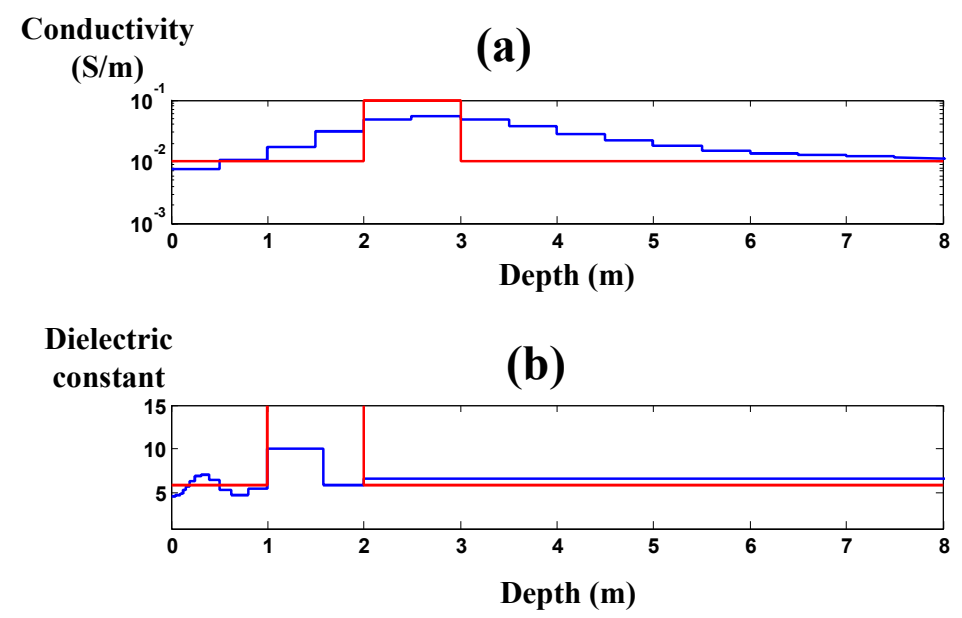

Fig. 3 (a) Inverted conductivity vs. depth. The red line is the simulated 1-D model labeled as MODEL 2 in Figure 2(a). (b) Inverted relative dielectric constant vs. depth. The red line represents the permittivity of the simulated MODEL 2. Note that the relative dielectric constant for the model between 1 and $2 \mathrm{~m}$ is 25 .

(a)
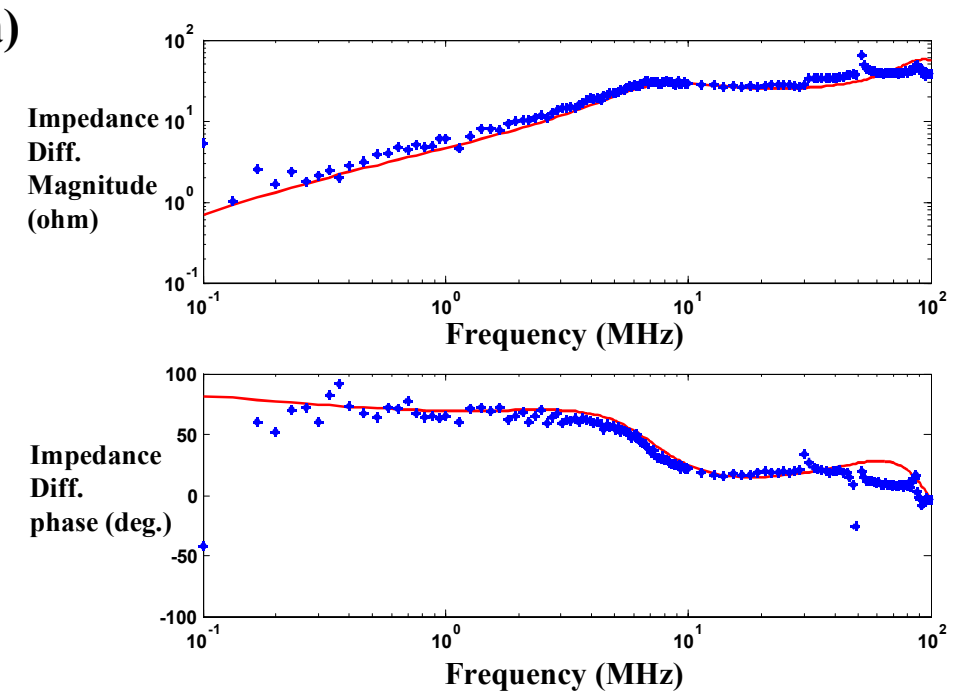

(b)

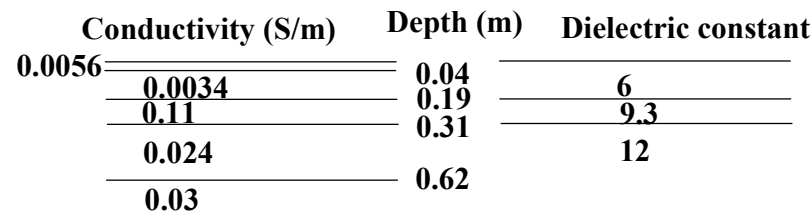

Fig. 4 (a) The measured impedance difference (blue asterisks) and simulated results (red lines) based on (b) the model derived from a resistivity sounding and an in situ TDR measurements. 